\title{
LOCALIZACIÓN DE QTLS PARA CARACTERES RELACIONADOS CON LA DOMESTICACIÓN DEL GIRASOL
}

\section{LOCALIZATION OF QTLS FOR DOMESTICATION-RELATED TRAITS IN SUNFLOWER}

\author{
Carlos J. Lozano Cavazos ${ }^{1}$, M. Humberto Reyes-Valdés; ${ }^{*}$ Francisco Castillo Reyes ${ }^{2}$, \\ Jesús Rodríguez de la $\mathrm{Paz}^{2}$, Octavio Martínez de la Vega ${ }^{3}$ y Alma P. García Villanueva ${ }^{2}$
}

\begin{abstract}
${ }^{1}$ Instituto Nacional de Investigaciones Forestales, Agrícolas y Pecuarias. Km. 6.5 Carretera Celaya-San Miguel de Allende. A. P. 112. 38110, Celaya, Guanajuato, México. ${ }^{2}$ Departamento de Fitomejoramiento, Universidad Autónoma Agraria Antonio Narro. 25315, Buenavista, Saltillo, Coahuila, México. ${ }^{3}$ Centro de Investigaciones y Estudios Avanzados-Irapuato, Instituto Politécnico Nacional. Km. 9.6 Carretera Irapuato-León. 36500, Irapuato, Guanajuato, México.

* Autor para correspondencia (mhreyes@uaaan.mx)
\end{abstract}

\section{RESUMEN}

El mejoramiento del girasol (Helianthus annuus L.) depende en gran medida de la introducción de diversidad genética proveniente de material silvestre. El objetivo de este trabajo fue construir un mapa de ligamiento basado en marcadores AFLP a partir de una

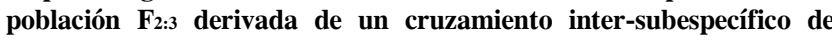
girasol (H. annuus var. macrocarpus $\mathrm{x} H$. annuus ssp. texanus Heiser), así como detectar posiciones de loci de caracteres cuantitativos (QTL) a través del enfoque analítico de mapeo por intervalos. Se evaluaron los siguientes caracteres contrastantes entre girasoles cultivados y silvestres, presumiblemente relacionados con la domesticación: altura de planta, número de capítulos (ramificación) y diámetro de capítulos por planta, número y peso de aquenios por capítulo, días a floración, días a madurez fisiológica y contenido de aceite de aquenios. La evaluación fenotípica se llevó a cabo en condiciones de campo, con un diseño de bloques incompletos con dos repeticiones. Se consideró un nivel de significancia estadística de amplitud genómica de 0.05 en la detección de los QTL. Para establecer los valores críticos de los estadísticos de prueba se hicieron pruebas de permutación sólo con los grupos de ligamiento con puntuaciones LOD (logaritmo de la razón de verosimilitudes) $>\mathbf{1 . 5}$. Además, para cada carácter se hizo un análisis de varianza por locus individual no ligado, con lo cual se identificaron cuatro loci no ligados que afectan el número de aquenios por capítulo y los días a madurez fisiológica, con $P<0.001$. Se identificó un QTL significativo con amplitud genómica de 0.017 para peso de aquenios que podría representar una región del genoma relacionada con la domesticación, y cinco QTLs posibles en cinco caracteres. Los QTLs detectados, que incluyen los hipotéticos, explicaron de 7.1 a $11.9 \%$ de la varianza fenotípica.

Palabras clave: Helianthus annuus L. var. macrocarpus, H. annuus L. ssp. texanus, mapa de ligamiento.

\section{SUMMARY}

Improvement of cultivated sunflower (Helianthus annuus L.) depends largely on the introduction of genetic diversity from wild species. The purpose of the present study was to construct a linkage map based on AFLP markers from an $F_{2: 3}$ population derived from a sunflower inter-subspecific cross $(H$. annuus var. macrocarpus $\mathbf{x}$ $H$. annuus ssp. texanus Heiser), as well as to detect quantitative trait loci (QTL) positions by interval mapping. The following contrasting traits between cultivated and wild sunflowers, putatively related to domestication, were evaluated: plant height, head number (branching), and head diameter per plant, number and weight of achenes per head, days to flowering, days to physiological maturity, and seed oil content. The phenotypic evaluation was carried out in field conditions, by using an incomplete block design with two replications. A genome-wide statistical significance of 0.05 was used to detect QTLs. To establish the empirical significance threshold values, permutation tests were performed only in linkage groups showing a LOD (logarithm of odds) score $>1.5$. Moreover, single marker-analyses using analysis of variance per unlinked individual locus for each trait were carried out, which identified four unlinked loci for number of achenes per head and days to physiological maturity with $P<0.001$. We identified one QTL with genome-wide significance of 0.017 for achene weight, which could be representative of a genome region produced by domestication, plus five putative QTLs in five characters. All the detected QTLs, including putative ones at the chosen significance level, explained from 7.1 to $11.9 \%$ of the phenotypic variance.

Index words: Helianthus annuus L. var. macrocarpus, H. annuus L. ssp. texanus, linkage map.

\section{INTRODUCCIÓN}

Recientemente, el girasol cultivado (Helianthus annuus L.) se ha considerado como una de las pocas especies domesticadas en el actual territorio de los Estados Unidos de América, sin embargo, el descubrimiento de restos de girasol domesticado con antigüedad aproximada de 400 años en San Andrés, Tabasco, México, implica un origen más antiguo y posiblemente independiente de domesticación, lo cual ha estimulado nuevas investigaciones sobre el origen geográfico del girasol domesticado (Lentz et al., 2001; Pope et al., 2001). Nueva evidencia de restos de girasol domesticado encontrados en la Cueva del Gallo, 
Morelos, México, con una antigüedad aproximada de 250 a 330 años a.C (Lentz et al., 2008), permita concluir la presencia precolombina de este cultivo en Mesoamérica.

Aunque no se conocen los antecesores del girasol cultivado, las poblaciones silvestres de Helianthus annuus que se distribuyen ampliamente en Norteamérica proveen de material de referencia para conocer qué caracteres de planta fueron modificados a través del proceso de domesticación, ya que todas ellas poseen, en contraste con el girasol, plantas ramificadas con capítulos y aquenios pequeños, así como semillas de bajo porcentajes de aceite, de manera que sirven como modelo biológico para entender la genética de la domesticación en esta especie.

En años recientes se ha utilizado la hibridación amplia para el mejoramiento del girasol, y se han utilizado especies silvestres de esta especie como fuentes de genes para características deseables (Seiler, 1992; Seiler y Rieseberg, 1997). El enfoque de mapas de ligamiento y mapeo de QTLs es una herramienta básica para el conocimiento de la genética de los caracteres cuantitativos. Existen varios mapas de ligamiento genético de girasol cultivado, generados a través de polimorfismos en la longitud de los fragmentos amplificados (AFLP) (Gedil et al., 2001; Langar et al., 2003; Kusterer et al., 2004). Estos mapas permiten el análisis de QTLs que posibilitan la detección de marcadores para la selección en mejoramiento genético (Staub et al., 1996).

No se han hecho estudios en poblaciones de mapeo híbridas biparentales entre girasoles cultivados y silvestres, para estudiar los caracteres contrastantes resultado de la domesticación. En este trabajo se plantearon los siguientes objetivos: 1) Construir un mapa de ligamiento genético de girasol basado en marcadores AFLP a partir de una población $\mathrm{F}_{2: 3}$ derivada de un cruzamiento entre girasol cultivado $(H$. annuus $\mathrm{L}$. var. macrocarpus, HA89) y silvestre (colecta Ac-8-2) (H. annuus L. ssp. texanus, Heiser); y 2) Detectar posiciones de loci de caracteres cuantitativos relacionados con la domesticación del girasol y estimar sus efectos genéticos.

\section{MATERIALES Y MÉTODOS}

Los cruzamientos inter-subespecíficos se efectuaron en un campo experimental localizado en Saltillo, Coah. $\left(25^{\circ}\right.$ 31' LN y $101^{\circ} 01^{\prime}$ LO, a $1743 \mathrm{msnm}$ ). El material genético utilizado fue la línea pública cultivada 'HA89' (H. annuus var. macroparpus), liberada por el Departamento de Agricultura de EE. UU. (USDA) y usada como estándar en investigaciones sobre girasol; esa línea se usó como progenitor femenino, y como progenitor masculino a un material silvestre colectado en
Saltillo, México e identificado como Ac-8-2 (H. annuus var. macrocarpus). Este último, representa una fuente de amplia variación genética, la cual puede utilizarse para el mejoramiento del girasol; además, es altamente contrastante con el girasol cultivado en los caracteres clave de domesticación ya que presenta capítulos, aquenios pequeños y es altamente ramificado.

Se obtuvieron plantas $F_{1}$, las cuales fueron sometidas a cruzamientos fraternos para obtener una población $\mathrm{F}_{2}$. La Figura 1 ilustra capítulos y semillas de progenitores y progenie. Luego se procedió a hacer autofecundaciones en esta última, para obtener líneas F3. El aislamiento del DNA genómico total se llevó a cabo con la técnica propuesta por Doyle y Doyle (1990). El análisis AFLP (Vos et al., 1995) se condujo con base en el protocolo IRDye Flourescent AFLP Kit ${ }^{\circledR}$ for Large Plant Genome Analysis LI-COR (Biosciences). Para la construcción del mapa de ligamiento se utilizó el programa JoinMap versión 3.0 (Van Ooijen y Voorrips, 2001).

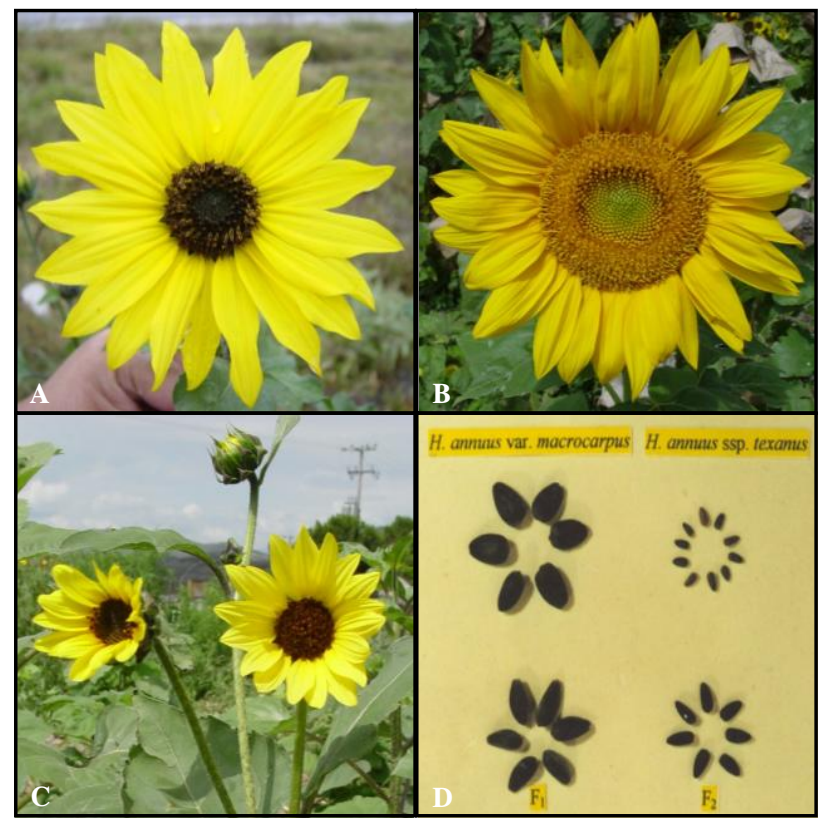

Figura 1. Capítulos y semillas de los progenitores de girasol silvestre y cultivado, así como de su progenie. A) Silvestre; B) Cultivado; C) Silvestre $\mathrm{x}$ cultivado (F1); D) Semilla de progenitores y progenie F1 y F2.

Los grupos de ligamiento se formaron con loci sin segregación distorsionada, con puntuaciones LOD (logaritmo de la razón de verosimilitudes) de 3.0 y frecuencia de recombinación máxima de 0.45 . La evaluación fenotípica replicada se hizo en un campo experimental en Navidad, N. L. (27 $04^{\circ} \mathrm{LN}$ y $100^{\circ} 56^{\prime}$ LO, a $1895 \mathrm{msnm}$ ), mediante un diseño con 135 familias anidadas en cuatro grupos acomodados en bloques, y evaluadas con dos repeticones. Tres de los grupos estuvieron compuestos por 34 familias, y uno por 33 
debido a pérdida de parcelas por condiciones de campo. Este tipo de arreglo se conoce como parcelas divididas anidadas (Kabelka et al., 2004) o bloques incompletos al azar (Oyervides et al., 1994). La distancia entre plantas, surcos y bloques fue de $0.70,0.80$ y $1.60 \mathrm{~m}$, respectivamente. Debido a la baja disponibilidad de semilla causada por autoincompatibilidad, solamente se pudieron establecer cuatro plantas por línea por repetición en cada parcela.

La altura de planta se midió cuando cada línea alcanzó $50 \%$ de apertura floral. El número de capítulos se contó cuando las plantas llegaron a madurez fisiológica. El diámetro de capítulo se midió en un muestreo aleatorio de cinco cabezuelas por planta, con medidas perpendiculares, y considerando solamente el promedio de éstas para el análisis de varianza. Estos mismos capítulos fueron autofecundados para la producción de aquenios. El peso, número y porcentaje de aceite de aquenios se evaluó en condiciones de laboratorio, en las cabezuelas colectadas para la evaluación del diámetro de capítulo. Los días a floración y días a madurez fisiológica se evaluaron en las etapas R5.5 y 9, respectivamente, según la descripción de etapas del crecimiento en girasol por Schneiter y Miller (1981). Los aquenios de cada capítulo muestreado fueron contabilizados y pesados para registrar el número y peso de aquenios. Para determinar el contenido de aceite en aquenios se utilizó el método para extracción de lípidos propuesto por Bligh y Dyer (1959). Para la detección de QTLs se consideró un nivel de significancia estadística de amplitud genómica igual a 0.05 , lo cual se determinó con el programa MapQTL ${ }^{\circledR}$ Versión 4.0 (Van Ooijen et al., 2002). Se estimaron los valores críticos de los estadísticos de prueba a través del método empírico propuesto por Doerge y Churchill (1996), basado en pruebas de permutación. Además, se realizó un análisis de varianza por locus marcador para cada carácter con el programa $\mathrm{R}$ versión 2.11.0 (R Development Core Team, 2010).

\section{RESULTADOS Y DISCUSIÓN}

La población de mapeo quedó constituida con 145 líneas $\mathrm{F}_{3}$ derivadas de $\mathrm{F}_{2}$. En el análisis de ligamiento se obtuvieron 295 bandas polimórficas, de las cuales se eliminaron 87 debido a que presentaron distorsión con respecto a la segregación mendeliana típica, con lo que se dispuso de 208 loci para realizar el análisis de ligamiento. La tasa de distorsión en la segregación fue alta y parece estar relacionada con causas diversas, como el incremento en la divergencia genética entre las líneas progenitoras (Quillet et al., 1995), irregularidades meióticas en la transmisión de los factores genéticos (Masuelii et al., 1995), selección gamética o cigótica (Kreike y Stiekema, 1997) y apareamiento preferencial entre genomas homólogos (Nybom et al., 2004). Por ejemplo, Paterson et al. (1988) reportaron $69 \%$ de marcadores con segregación distorsionada en una población $\mathrm{F}_{2}$ derivada del híbrido interespecífico Lycopersicon esculentum $\mathrm{x}$ Lycopersicon chmielewskii.

De los 208 loci utilizados para el mapa de ligamiento, sólo $19.23 \%$ (40 loci) se aglutinó en 17 grupos de ligamiento (Figura 2), con una puntuación LOD mínima de 3.0 y una frecuencia de recombinación máxima de 0.45 . Lo anterior produjo un mapa con baja densidad de marcadores. En el análisis fenotípico se observaron diferencias significativas $(\mathrm{P} \leq 0.001)$ entre familias dentro de grupos para altura de planta, número de capítulos, diámetro de capítulos, peso y contenido de aceite de aquenios (Cuadro 1). De acuerdo con lo anterior, se infiere la existencia de variación genética en la población. El contenido de aceite de aquenios fluctuó de 20.16 a $43.10 \%$. En este contexto, Burke et al. (2005) reportaron un contenido de aceite de 40 y $26 \%$ en girasol cultivado y silvestre, respectivamente.

La variación genética en estos caracteres se atribuye a los efectos de la selección en el proceso de domesticación de esta especie, por el contraste entre materiales silvestres y cultivados, que dan origen a una alta segregación en la progenie del cruzamiento. En especial, el proceso de domesticación inicial del girasol incrementó el peso de los aquenios y el diámetro de los capítulos, y luego, en épocas más recientes, el interés en la producción de aceite de girasol se tradujo en estrategias de mejoramiento genético que causaron la marcada diferencia en la concentración de este producto natural entre poblaciones domésticas y silvestres de girasol.

Cuadro 1. Cuadrados medios para seis caracteres relacionados a la domesticación del girasol en una población segregante F2:3.

\begin{tabular}{|c|c|c|c|c|c|c|c|}
\hline \multirow[t]{2}{*}{ Fuente de variación } & \multirow[b]{2}{*}{$\mathrm{gl}$} & \multicolumn{6}{|c|}{ Caracteres $^{\dagger}$} \\
\hline & & $\mathrm{AP}$ & $\mathrm{NC}$ & $\mathrm{DC}$ & NA & PA & CA \\
\hline Repeticiones & 1 & $0.0519 \mathrm{~ns}$ & $178.3 \mathrm{~ns}$ & $0.1598 \mathrm{~ns}$ & $86.81 \mathrm{~ns}$ & $0.129 \mathrm{~ns}$ & $0.08397 * * *$ \\
\hline Grupos & 3 & $0.1214 * * *$ & $3695.1 * * *$ & $1.6598 \mathrm{~ns}$ & $312.23 \mathrm{~ns}$ & $0.311 \mathrm{~ns}$ & $0.00928 * * *$ \\
\hline Familias/grupos & 131 & $0.0895 * * *$ & $2858.2 * * *$ & $2.4241 * * *$ & $986.89 *$ & $1.2102 * * *$ & $0.00465 * * *$ \\
\hline Error residual & 134 & 0.0197 & 348.4 & 1.229 & 746.13 & 0.1756 & 0.00074 \\
\hline
\end{tabular}

${ }^{\dagger} \mathrm{gl}=$ grados libertad; $\mathrm{AP}=$ altura de planta $(\mathrm{m}) ; \mathrm{NC}=$ número de capítulos; $\mathrm{DC}=$ diámetro de capítulos $(\mathrm{cm}) ; \mathrm{NA}=\mathrm{número}$ de aquenios; $\mathrm{PA}=$ peso de aquenios $(\mathrm{g}), \mathrm{CA}=$ contenido de aceite de aquenios $(\%){ }^{*}=\mathrm{P} \leq 0.05, * * \mathrm{P} \leq 0.01 \mathrm{y}, * * * \mathrm{P} \leq 0.001 \mathrm{de}$ probabilidad, respectivamente; ns $=$ no significativo. 

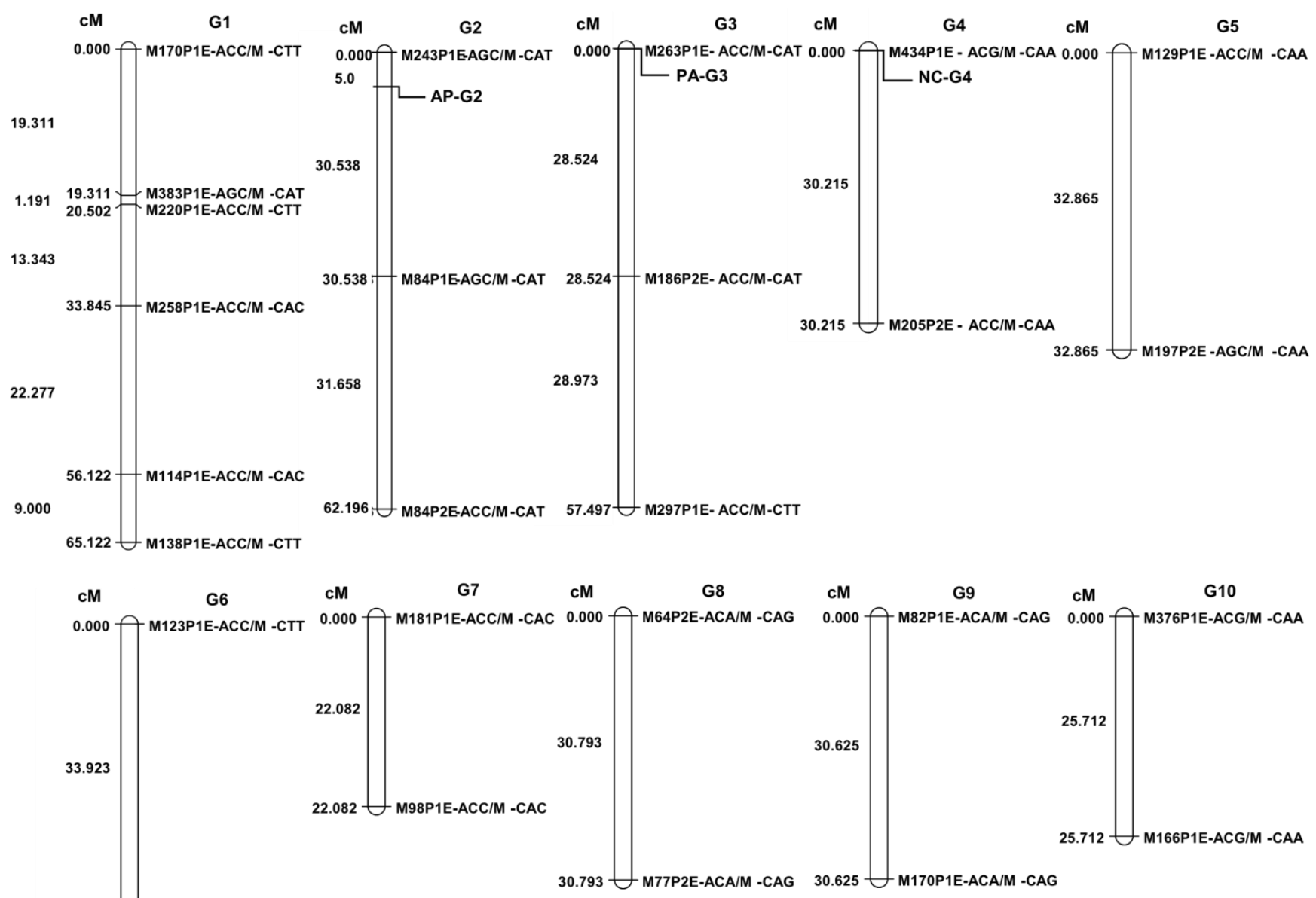

$33.923 \bigcup_{\text {M181P1E-ACC/M -CTT }}$
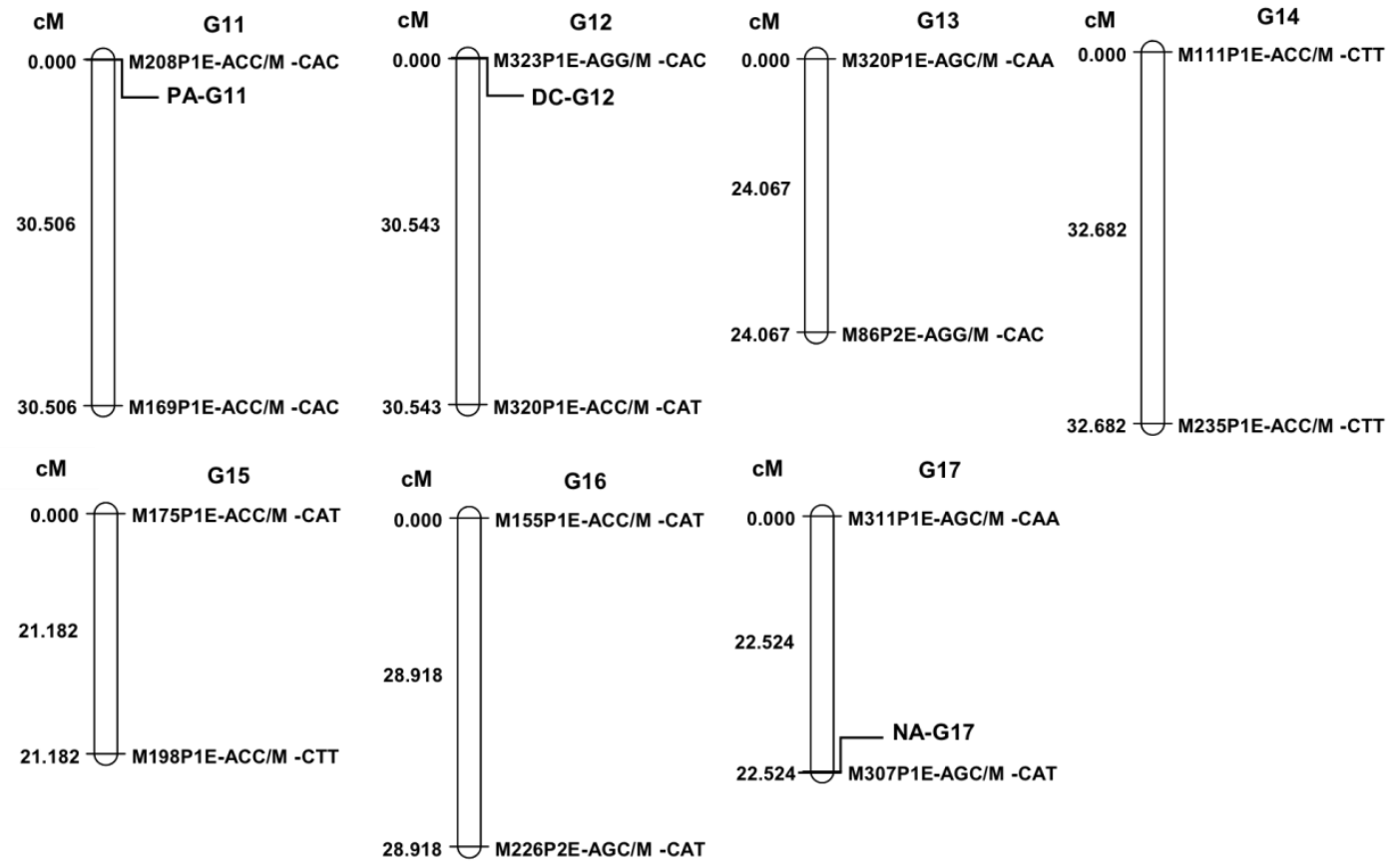

Figura 2. Mapa de ligamiento de girasol obtenido en una población $\mathbf{F}_{2: 3}$, derivada del cruzamiento entre girasol cultivado (Helianthus annuus L. var. macrocarpus, HA89) y silvestre (Helianthus annuus L. ssp. texanus, Heiser) con marcadores AFLP. 
Dicho contraste originó la variación aquí observada en el contenido de aceite entre las líneas recombinantes. El polimorfismo que exhibe esta población indica que es un material biológico útil para estudiar la genética de las características centrales de los girasoles cultivados, sobre todo a través del análisis de QTLs.

Se detectó un QTL con significancia de amplitud genómica de 0.017 para peso de aquenios (PA-G11), el cual está asociado al locus marcador M208P1E-ACC/M$\mathrm{CAC}$, con un efecto aditivo estimado de $0.206 \mathrm{~g}$ que explicó $10.2 \%$ de la varianza fenotípica (Figuras 3B y C); este QTLs podría representar una región cromosómica relacionada con la domesticación del girasol, ya que el alelo del material cultivado tiene un efecto positivo. Además, se detectaron cinco QTL posibles (con significancia de amplitud de grupo de ligamiento $<0.05$, pero con valor de amplitud genómica $>0.05$ ), que afecta la altura de planta, número y diámetro de capítulos, número y peso de aquenios (Cuadro 2). El efecto aditivo de cada QTL identificado se determinó al considerar el efecto como positivo cuando la media estimada del carácter cuantitativo asociado a los alelos del progenitor cultivado ('HA89') fue mayor que la media de los alelos silvestres (Cuadro 2; Figuras 3B y D) y viceversa, por lo que la direccionalidad de los efectos alélicos depende en gran parte del fondo genético.

Cuadro 2. QTLs de caracteres relacionados con la domesticación del girasol en una población segregante F2:3, identificados mediante mapeo por intervalos.

\begin{tabular}{llcccrcrcr}
\hline Carácter & \multicolumn{1}{c}{ QTL $^{\dagger}$} & $\begin{array}{c}\text { Posición } \\
(\mathrm{cM})^{\uparrow}\end{array}$ & $\begin{array}{c}\text { Locus marcador } \\
\text { flanqueante }\end{array}$ & $\begin{array}{c}\text { Puntuación } \\
\text { LOD }\end{array}$ & PVF & AG & $\begin{array}{c}\text { AC } \\
\text { aditivos }\end{array}$ \\
\hline AP & AP-G2 & 5.0 & M243P1E-AGC/M-CAT & 1.94 & 10.3 & 0.204 & 0.012 & 0.095 \\
NC & NC-G4 & 0.0 & M434P1E-ACG/M-CAA & 2.24 & 9.0 & 0.085 & 0.005 & -8.211 \\
DC & DC-G12 & 0.0 & M323P1E-AGG/M-CAC & 1.62 & 7.1 & 0.170 & 0.010 & -0.453 \\
NA & NA-G17 & 22.5 & M307P1E-AGC/M-CAT & 1.53 & 8.5 & 0.187 & 0.011 & 8.925 \\
PA & PA-G3 & 0.0 & M263P1E-ACC/M-CAT & 1.92 & 11.9 & 0.085 & 0.005 & 0.239 \\
PA & PA-G11 & 0.0 & M208P1E-ACC/M-CAC & 1.89 & 10.2 & 0.017 & 0.001 & 0.206 \\
\hline
\end{tabular}

$\mathrm{AP}=$ altura de planta $(\mathrm{m}) ; \mathrm{NC}=$ número de capítulos; $\mathrm{DC}=$ diámetro de capítulos $(\mathrm{cm})$; $\mathrm{NA}=$ número de aquenios; $\mathrm{PA}=$ peso de aquenios $(\mathrm{g})$. ${ }^{\dagger}$ La descripción de cada QTL está de acuerdo con la abreviación de cada carácter y con el grupo de ligamiento en el mapa genético. ${ }^{\circledR}$ Posición del QTL respecto al primer locus marcador flanqueante. Puntuación LOD = logaritmo de la razón de verosimilitudes; PVF = porcentaje de la varianza fenotípica explicada por el QTL; AG = significancia estadística de amplitud genómica; AC = significancia estadística de amplitud cromosómica.
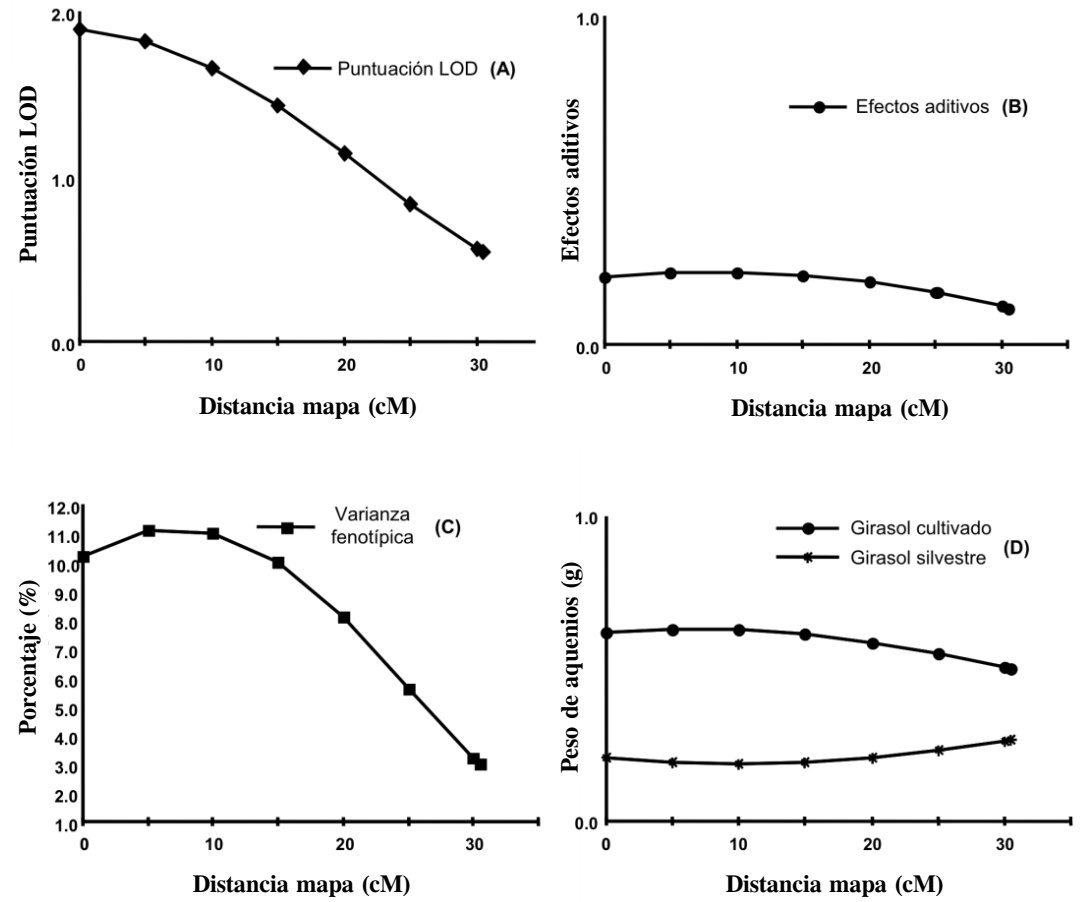

Figura 3. Perfiles de los QTL con significancia de amplitud genómica para peso de aquenios en el grupo de ligamiento 11, denominado PA-G11. (A) Puntuación LOD (logaritmo de la razón de verosimilitudes); (B) Efectos aditivos; (C) Varianza fenotípica explicada por el QTL; y (D) Media estimada de la distribución del carácter peso de aquenios. 
El análisis de marcadores individuales identificó 96 loci significativos de los 168 loci polimórficos no ligados al mapa genético asociados a los ocho caracteres evaluados, de los cuales uno se encuentra potencialmente asociado con el número de aquenios (M120P1E-AGC/M-CAA) y tres con los días a madurez fisiológica (M290P1EACC/M-CTT, M486P2E-ACC/M-CTT, M177P1E$\mathrm{ACC} / \mathrm{M}-\mathrm{CTT})$ con $\mathrm{P}<0.001$.

En el presente trabajo se obtuvo el primer mapa de girasol a partir de un cruzamiento inter-subespecífico entre girasol cultivado y silvestre, con distancia promedio entre marcadores de $14.65 \mathrm{cM}$. Este mapa es útil para el análisis de los caracteres relacionados con la domesticación, ya que los progenitores son altamente contrastantes para los mismos. Se observó variación genética en la población, y se identificó un QTL significativo en el grupo de ligamiento 11 y cinco QTLs a nivel hipotético. Los seis QTLs detectados explicaron de 7.1 a $11.9 \%$ de la varianza fenotípica para los caracteres evaluados.

La localización de los QTLs fue afectada de manera considerable ya que los caracteres cuantitativos suelen tener gran interacción genotipo $\mathrm{x}$ ambiente, $\mathrm{y}$ no fue posible evaluar más localidades por escasez de semilla. Sin embargo, este trabajo contribuye de manera preliminar al conocimiento de la arquitectura genética de los caracteres cuantitativos de importancia en el proceso de domesticación de esta especie. La adición de otros tipos de marcadores, como los basados en microsatélites, a este grupo de ligamiento aumentaría su poder de resolución, si se implementa una estrategia para incrementar la cantidad de semilla de las líneas recombinantes y así hacer las evaluaciones fenotípicas con muestras más grandes y en varios ambientes, lo que permitirá obtener un conocimientio más profundo de la genética de la domesticación del girasol.

\section{CONCLUSIONES}

Se desarrolló una población de líneas de girasol a partir del cruzamiento entre un material cultivado y un silvestre, la cual presenta polimorfismo genético para caracteres relacionados con la domesticación de $H$. annuus. A partir de esta población se construyó un mapa de ligamiento de AFLP de baja densidad, que constituye una base para el desarrollo de un mapa saturado y el estudio más profundo de la genética de los caracteres contrastantes entre girasoles silvestres y cultivados. Se detectó un QTL significativo para tamaño de aquenios, que podría representar una región del genoma del girasol relacionada con su domesticación.

\section{AGRADECIMIENTOS}

Este trabajo fue financiado a través de un proyecto de investigación básica del Consejo Nacional de Ciencia y Tecnología (CONACYT) y por la Universidad Autónoma Agraria Antonio Narro. Asimismo, el CONACYT proveyó la beca de estudios de postgrado del Dr. Carlos Javier Lozano Cavazos, para la consecución de la tesis de la cual se derivó esta investigación.

\section{BIBLIOGRAFÍA}

Bligh E G, W J Dyer (1959) A rapid method of total lipid extraction and purification. Can. J. Biochem. Physiol. 37:911-917.

Burke J M, S J Knapp, L H Rieseberg (2005) Genetic consequences of selection during the evolution of cultivated sunflower. Genetics 171:1933-1940.

Doerge R W, G A Churchill (1996) Permutation tests for multiple loci affecting a quantitative character. Genetics 142:285-294.

Doyle J J, J L Doyle (1990) Isolation of plant DNA from fresh tissue. Focus 12:13-15.

Gedil M A, C Wye, S Berry, B Segers, J Peleman, R Jones, A Leon, M B Slabaugh, S J Knapp (2001) An integrated restriction fragment length polymorphism-amplified fragment length polymorphism linkage map for cultivated sunflower. Genome 44:213-221.

Kabelka E A, B W Diers, W R Fehr, A R LeRoy, I C Baianu, T You, D J Neece, R L Nelson (2004) Putative alleles for increased yield from soybean plant introductions. Crop Sci. 784-791.

Kreike C M, W J Stiekema (1997) Reduced recombination and distorted segregation in a Solanum tuberosum $(2 \mathrm{x}) \mathrm{x} S$. spegazzinii (2x) hybrid. Genome 40:180-187.

Kusterer B, B Rozynek, L Brahm, M Prüfe, S Tzigos, R Horn, W Friedt (2004) Construction of a genetic map and localization of major traits in sunflower (Helianthus annuus L.). Helia 27:1524.

Langar K, M Lorieux, E Desmarais, Y Griveau, L Gentzbittel, A Bervillé (2003) Combined mapping of DALP and AFLP markers in cultivated sunflower using F9 recombinant inbred lines. Theor. Appl. Genet. 106:1068-1074.

Lentz D L, M D Pohl, K O Pope, A R Wyatt (2001) Prehistoric sunflower (Helianthus annuus L.) domestication in Mexico. Econ. Bot. 55:370-376.

Lentz D L, M D Pohl, J L Alvarado, S Tarighat, R Bye (2008) Sunflower (Helianthus annuus L.) as a pre-Columbian domesticate in Mexico. Proc. Nat. Acad. Sci. USA 105:62326237.

Masuelli R W, E Y Tanimoto, C R Brown, L Comai (1995) Irregular meiosis in a somatic hybrid between $S$. bulbocastanum and $S$. tuberosum detected by species-specific PCR markers and cytological analysis. Theor. Appl. Genet. 91:401-408.

Nybom H, G D Esselink, G Werlemark, B Vosman (2004) Microsatellite DNA marker inheritance indicates preferential pairing between two highly homologous genomes in polyploidy and hemisexual dog-roses, Rosa L. Sect. Caninae DC. Heredity 92:139-150.

Oyervides G A, J M Mariaca P, H De León C, M Reyes V (1993) Estimación de parámetros genéticos en una población de maíz tropical. Agron. Mesoam. 4:30-35.

Paterson A H, E S Lander, J D Hewitt, S Peterson, S E Lincoln, S D Tanksley (1988) Resolution of quantitative traits into mendelian factors by using a complete linkage map of restriction fragment length polymorphisms. Nature 335:721-726. 
Pope K O, M E D Pohl, J G Jones, D L Lentz, C von Nagy, F J Vega, I R Quitmyer (2001) Origin and environmental setting of ancient agriculture in the lowlands of Mesoamerica. Science 292:1370-1373.

Quillet M C, N Madjidian, Y Griveau, H Serieys, M Tersac, M Lorieux, A Bervillé (1995) Mapping genetic factors controlling pollen viability in an interspecific cross in Helianthus sect. Helianthus. Theor. Appl. Genet. 91:1195-1202.

R Development Core Team (2010) R: A Language and Environment for Statistical Computing. $\mathrm{R}$ Foundation for Statistical Computing, Vienna, Austria. Disponible en: http://www.Rproject.org. (Junio, 2010).

Schneiter A A, J F Miller (1981) Description of sunflower growth stages. Crop Sci. 21:901-903.

Seiler G J (1992) Utilization of wild sunflower species for the improvement of cultivated sunflower. Field Crops Res. 30:195 230 .
Seiler G J, H L Reiseberg (1997) Systematics, origin, and germplasm resources of the wild and domesticated sunflower. In: Sunflower Technology and Production. A A Schneiter (ed). ASA, CSSA, SSSA, Medison, Wisconsin, USA. pp:21-65.

Staub J E, F C Serquen, M Gupta (1996) Genetic markers, map construction and their application in plant breeding. Hortscience 31:729-741.

Van Ooijen J W, R E Voorrips (2001) JoinMap ${ }^{\circledR}$ 3.0: Software for the calculation of genetic linkage maps. Plant Research International, Wageningen, The Netherlands.

Van Ooijen J W, M P Boer, R C Jansen, C Maliepaard (2002) MapQTL ${ }^{\circledR}$ 4.0: Software for the calculation of QTL positions on genetic maps. Plant Research International, Wageningen, The Netherlands.

Vos P, R Hogers, M Bleeker, M Reijans, T Van de Lee, M Hornes, A Frijters, J Pot, J Peleman, M Kuiper, M Zabeau (1995) AFLP: a new technique for DNA fingerprinting. Nucleic Acids Res. 23:4407-4414. 